\title{
A Patient with a Large Basilar Artery Aneurysm in Whom Coil Embolization Was Performed by Protruding an LVIS into the Aneurysmal Neck in a Barrel-like Shape and Preserving a Branch Vessel
}

Tomoyuki Yamashita, Hiroyuki Ikeda, Ryotaro Otsuka, Sadaharu Torikoshi, Noritaka Sano, Makoto Hayase, and Hiroki Toda

Objective: We report a case of a low-profile visualized intraluminal support device (LVIS) being deployed and protruded into an aneurysmal neck in a barrel-like shape to perform dense coil embolization while preserving the branch vessel from the aneurysmal dome in order to prevent aneurysmal enlargement.

Case Presentation: A 74-year-old woman had a recurrent large cerebral aneurysm at the bifurcation of the basilar artery and the left superior cerebellar artery (SCA). Therefore, an LVIS was deployed from the left posterior cerebral artery to the basilar artery and protruded into the aneurysmal neck in a barrel-like shape to increase its metal coverage ratio. As the barrel-shaped protruding LVIS served as a scaffold to support the coils, dense coil embolization was performed while preserving the SCA branching from the aneurysmal dome. Images obtained at 6 months and 1 year after the embolization confirmed preservation of the SCA and prevention of aneurysmal enlargement.

Conclusion: Protruding the LVIS into an aneurysmal neck in a barrel-like shape is a technique that may help preserve the branch vessel and facilitate dense coil embolization.

Keywords > LVIS, stent, aneurysm, bulging, coil embolization

\section{Introduction}

Barrel, bulging, and shelf methods of coil embolization in which a low-profile visualized intraluminal support junior device (LVIS Jr.) stent (Terumo Corporation, Tokyo, Japan) is protruded into the origin of a branch vessel from the cerebral aneurysmal neck to preserve the vessel have been reported. ${ }^{1-3)}$ A similar procedure may be achieved using a similar braided stent, an LVIS stent (Terumo Corporation),

Department of Neurosurgery, Fukui Red Cross Hospital, Fukui, Fukui, Japan

Received: February 27, 2020; Accepted: May 26, 2020 Corresponding author. Hiroyuki Ikeda. Department of Neurosurgery, Kurashiki Central Hospital, 1-1-1, Miwa, Kurashiki, Okayama 710-8602, Japan

Email: rocky@kuhp.kyoto-u.ac.jp

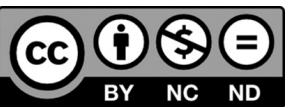

This work is licensed under a Creative Commons Attribution-NonCommercialNoDerivatives International License.

(C)2020 The Japanese Society for Neuroendovascular Therapy whose wire count, metal coverage rate, and diameter are higher/larger than those of an LVIS Jr. We report a patient in whom dense coil embolization of a recurrent large cerebral aneurysm was performed while preserving a branch vessel from the aneurysmal dome by protruding an LVIS into the aneurysmal neck in a barrel-like shape such that the metal coverage rate was high, preventing enlargement of the aneurysm.

\section{Case Presentation}

The patient was a 74-year-old, right-handed woman. She had a history of hypertension, dyslipidemia, and unruptured multiple cerebral aneurysms. She had undergone coil embolization of a cerebral aneurysm at the left internal carotid artery-superior hypophyseal artery bifurcation 18 years previously. Furthermore, she had undergone clipping of a distal right anterior cerebral artery aneurysm under craniotomy 9 years previously, in addition to the same procedure for a left middle cerebral artery aneurysm 8 years previously. To treat a cerebral aneurysm at the bifurcation 

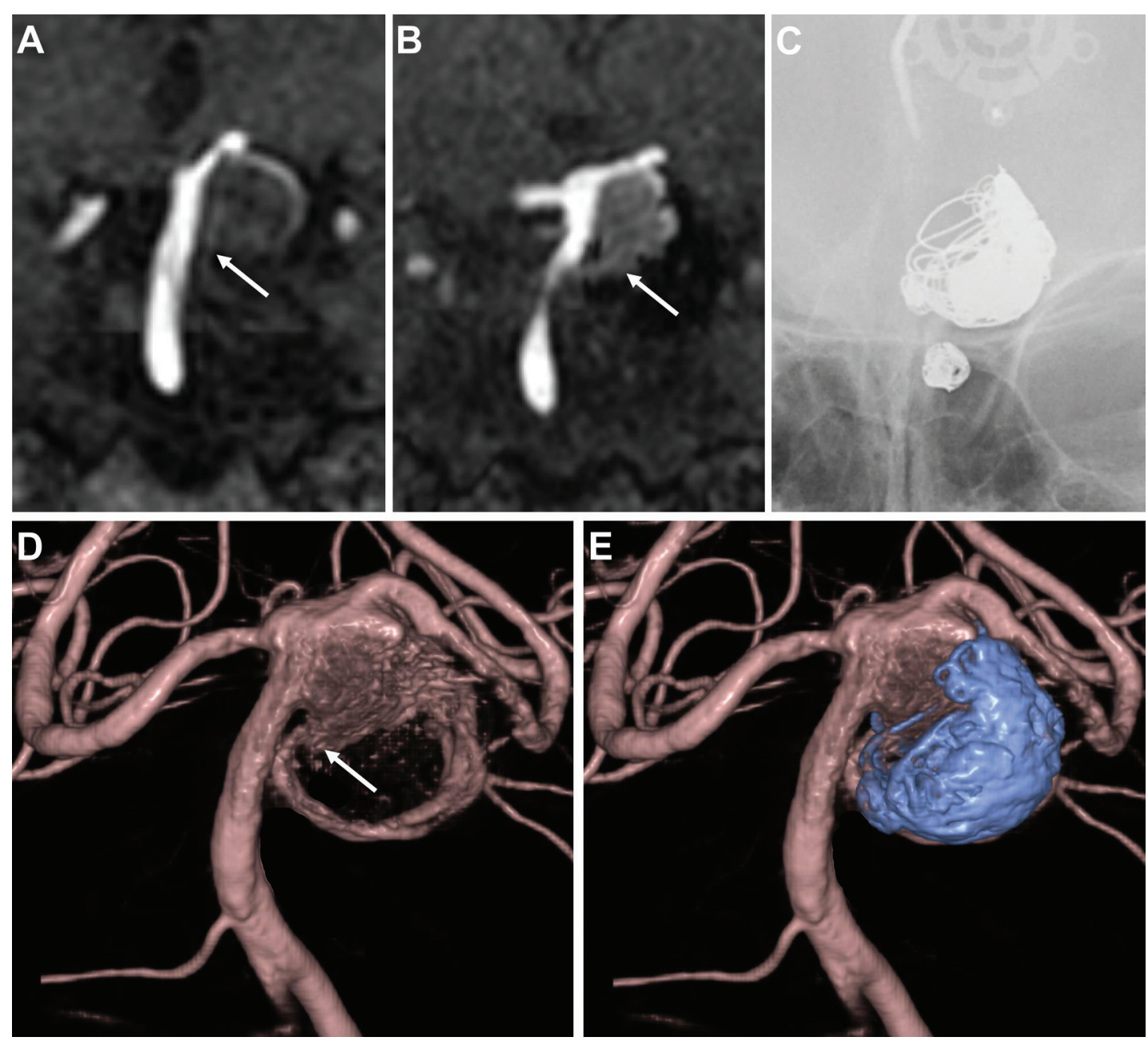

Fig. 1 Imaging findings before treatment. (A) Coronal section of an original MRA image before coil embolization 8 years previously. The left SCA (arrow) had branched from the aneurysmal neck. (B) Coronal section of an original MRA image. The left SCA (arrow) had branched from the aneurysmal dome. (C) Frontal view of cephalic X-ray. Coil compaction was observed. (D) Three-dimensional cerebral angiography. Left SCA (arrow). (E) Image in which a three-dimensional cerebral angiogram was fused with a coil mass (blue area) on initial treatment. SCA: superior cerebellar artery

between the basilar and left superior cerebellar arteries, she had undergone coil embolization via a simple technique 8 years previously (Fig. 1A). For the purpose of follow-up, she had been referred to our hospital 3 years previously. However, cephalic MRA revealed a serial increase in the blood flow space of a cerebral aneurysm at the basilar artery-left superior cerebellar artery (SCA) bifurcation. Cephalic X-ray revealed coil compaction (Fig. 1B and 1C). On cerebral angiography, the blood flow space of the aneurysm measured $9.8 \mathrm{~mm}$ in neck diameter, $10.0 \mathrm{~mm}$ in height, and $11.0 \times 9.3 \mathrm{~mm}$ in dome size. The diameter of the left posterior cerebral artery was $2.1 \mathrm{~mm}$ and that of the basilar artery was $2.9 \mathrm{~mm}$. The left SCA was sharply branched from the aneurysmal dome toward the basilar artery (Fig. 1D and 1E). There was no neurological abnormality related to compression of the cerebral aneurysm, but the aneurysm size, which had serially increased, exceeded $10 \mathrm{~mm}$, suggesting that the risk of aneurysmal rupture was relatively high. Neuroendovascular treatment was performed based on the patient's and her family's wishes.

\section{Neuroendovascular treatment}

Aspirin at $100 \mathrm{mg}$ /day and clopidogrel at $75 \mathrm{mg} /$ day were orally administered for 2 weeks before neuroendovascular treatment. Under general anesthesia, systemic heparinization was conducted and an 8-Fr guiding catheter was inserted to the V2 portion of the left vertebral artery through the right femoral artery. As guiding-catheter insertion induced stasis of left vertebral artery blood flow, left vertebral artery perfusion was persistently conducted using the vertebral artery flow reversal method. ${ }^{4)}$ Using a 3.4-Fr TACTICS $130 \mathrm{~cm}$ (Technocrat Corporation, Aichi, Japan) 

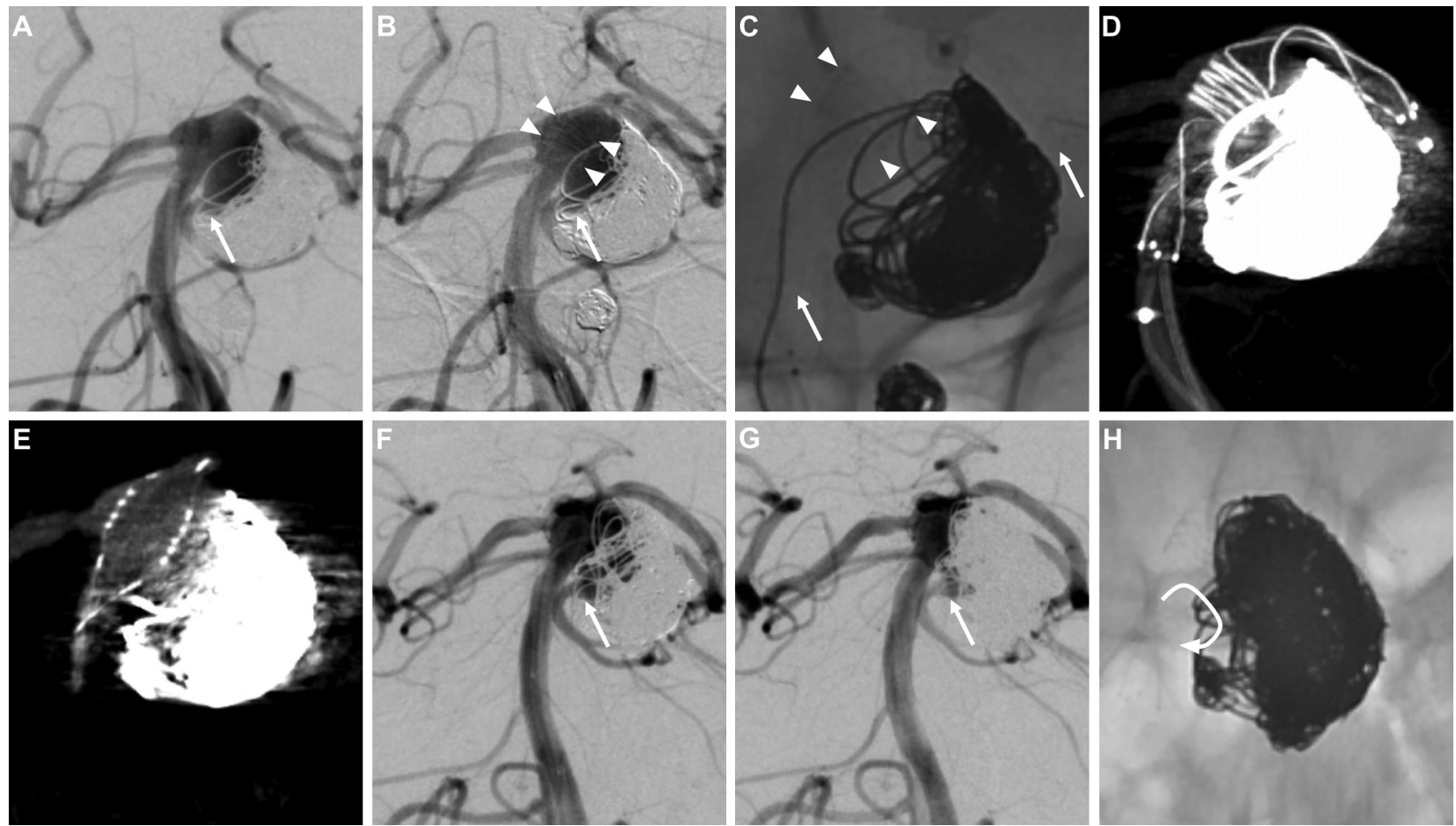

Fig. 2 Imaging findings during embolization (frontal view). (A) Cerebral angiography before treatment. The left SCA (arrow) had branched from the aneurysmal dome. (B) Cerebral angiography after LVIS deployment. The LVIS protruded into the aneurysmal neck in a barrel-like shape (arrowheads). The LVIS could not cover the origin of the left SCA (arrow). (C) Fluoroscopy after LVIS deployment. Both sides of an LVIS (arrows) protruded in a barrel-like shape (arrowheads). (D and E) Three-dimensional (D) and coronal (E) sections of cone-beam CT with contrast medium diluted at a ratio of 5. (F) Cerebral angiography after first-coil insertion. Framing to preserve the blood flow space of the left SCA (arrow) was achieved. (G) Cerebral angiography after coil embolization. A coil mass involving preservation of the blood flow space of the left SCA (arrow) was formed. (H) Coil mass after coil embolization. A coil mass was formed in order for the coil to not fill the blood flow space of the left SCA (arrow). LVIS: low-profile visualized intraluminal support device; SCA: superior cerebellar artery

as a distal access catheter through the guiding catheter, a Headway $21156 \mathrm{~cm}$ (Terumo Corporation) was guided into the left posterior cerebral artery. A C-steam-shaped Excelsior SL-10 (Stryker, Kalamazoo, MI, USA), which had been prepared in accordance with the shapes of the basilar artery and aneurysm, was guided into the aneurysm and inserted from the inflow zone toward the outflow zone. Through the Excelsior SL-10, two loops of an Axium Prime Frame $9 \times 30 \mathrm{~cm}$ (Medtronic, Minneapolis, MN, USA) were inserted into the aneurysm, and an LVIS $5.5 \times$ $33 \mathrm{~mm}$ was deployed using "wire-push" operations from the proximal P2 portion of the left posterior cerebral artery. At the aneurysmal neck, the LVIS was pushed out of the Headway 21 using "wire-push" operations and shortened using "system-push" operations. By repeating this, the LVIS was protruded into the aneurysmal neck in a barrel-like shape in order for the metal coverage rate to be high. In the basilar artery proximal to the aneurysm, an LVIS was deployed using "wire-push" operations. Cerebral angiography and cone-beam CT revealed that the LVIS had protruded into the aneurysmal neck in a barrel-like shape, with a high metal coverage rate (Fig. 2A-2E). As the first coil, an Axium Prime Frame $9 \times 30 \mathrm{~cm}$ was used. The coil was supported by the LVIS protruding in a barrel-like shape, and framing in which the SCA was preserved was relatively readily achieved by repeatedly inserting and pulling the coil through the jailed microcatheter (Fig. 2F). Using an Axium Prime Frame $8 \times 30 \mathrm{~cm}$, Target $360 \mathrm{Soft}$ $7 \times 30 \mathrm{~cm}$ (Stryker), and Target $360 \mathrm{Soft} 7 \times 15 \mathrm{~cm}$, filling was performed in the framing coil in which the SCA was preserved. The Excelsior SL-10 was pushed back to the inflow zone, and filling and finishing of the inflow zone were conducted using two Target 360 Ultra $5 \times 15 \mathrm{~cm}$ and a Galaxy G3 XSFT $4 \times 8 \mathrm{~cm}$ (Cerenovus, Irvine, CA, USA). Cerebral angiography confirmed preservation of the SCA and complete occlusion of the aneurysm, excluding the bifurcation of the SCA (Fig. $\mathbf{2 G}$ and $\mathbf{2 H}$ ).

\section{Postoperative course}

After surgery, there was no neurological abnormality. No ischemic lesion was noted on MRI the day after surgery. MRA confirmed preservation of the left SCA (Fig. 3A). 

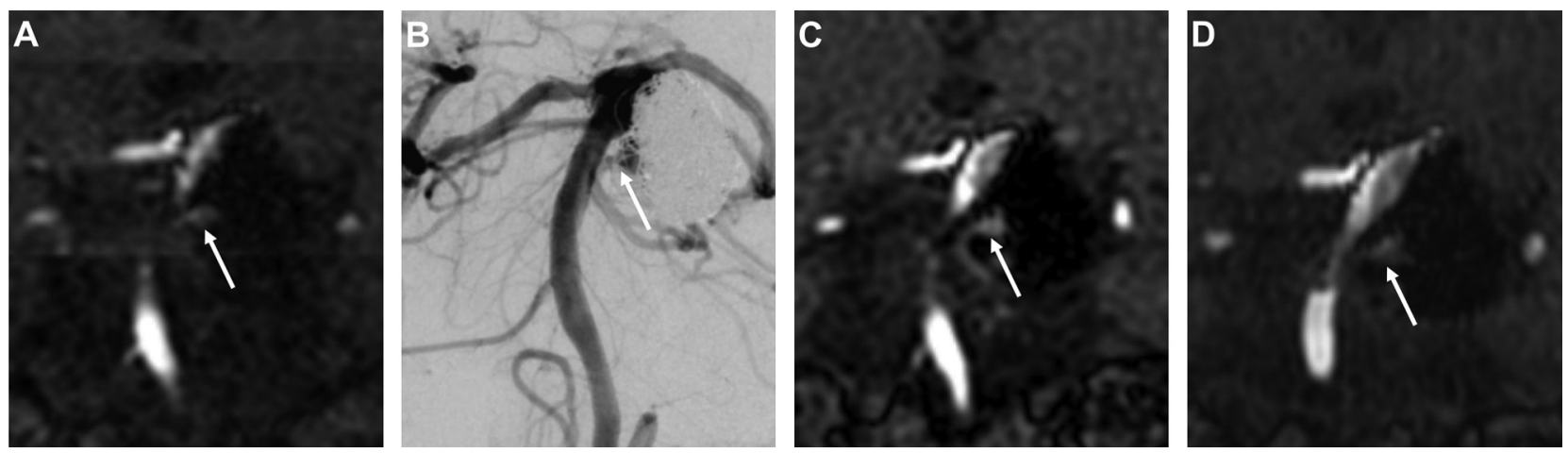

Fig. 3 Imaging findings after embolization. (A) Coronal section of an original MRA image the day after embolization. The left SCA (arrow) was visualized. (B-D) Cerebral angiography 6 months after embolization (B), and MRA (coronal sections of original images) 6 months (C) and 1 year (D) after embolization confirmed visualization of the left SCA (arrow) and absence of aneurysmal enlargement. SCA: superior cerebellar artery

The course was favorable, and the patient was discharged 4 days after surgery. The oral administration of aspirin and clopidogrel was continued. MRA and cerebral angiography 6 months after surgery confirmed preservation of the left SCA and absence of aneurysmal enlargement (Fig. 3B and $\mathbf{3 C}$ ). The administration of clopidogrel was completed, and aspirin therapy was continued. MRA 1 year after surgery reconfirmed preservation of the left SCA and absence of aneurysmal enlargement (Fig. 3D).

\section{Discussion}

When performing coil embolization of wide-necked aneurysms with a branch vessel, coil deviation into parent or branch vessels can be prevented by covering the aneurysmal neck and origin of the branch vessel with an LVIS Jr. stent using the barrel, bulging, and shelf methods. ${ }^{1-3)} \mathrm{Du}$ and Shankar performed coil embolization of wide-necked aneurysms with a branch vessel using the shelf technique in 8 patients and reported that the procedure was successful in all with no morbidity or mortality, suggesting the feasibility and safety of the shelf technique. ${ }^{1)}$ Thus, it is not necessary to insert a microwire or -catheter into a branch vessel to be preserved. The insertion of a single stent facilitates dense coil embolization and branch-vessel preservation; therefore, the procedure may be relatively easy and highly safe. On the other hand, the origin of a branch vessel may not completely be covered by a stent, and coil embolization at a working angle at which a branch vessel is isolated is necessary to confirm coil deviation. ${ }^{1,2)}$ To dilate an LVIS Jr. at maximum using "wire-push" and "system-push" operations, complete stent dilation and the absence of torsion should be confirmed using cone-beam CT after stent deployment. ${ }^{1)}$
In the present case, the main purpose of treatment was to preserve the SCA branching from the aneurysmal dome and inhibit growth of the aneurysm. The recanalization rate after coil embolization of large or giant aneurysms is high, but stent-assisted coil embolization may reduce this rate ${ }^{5}$; therefore, stent-assisted coil embolization was selected. To preserve branch vessels, highly difficult procedures, such as the Y-stent, ${ }^{6}$ kissing Y-stent, ${ }^{7)} \mathrm{T}$-stent, ${ }^{8)}$ and stent and balloon ${ }^{9)}$ methods, have been reported. However, in the present case, the branching angle of the SCA from the basilar artery was sharp, and wire/catheter guiding into the SCA was considered to be difficult. Therefore, we planned to preserve a branch vessel by protruding a stent into the aneurysmal neck, as described for the barrel, bulging, and shelf methods. A stent with a diameter larger than that of an LVIS Jr., which dilates to $3.7 \mathrm{~mm}$ in maximum diameter, was considered to be necessary for preservation of a branch vessel from the aneurysmal dome. Thus, we selected an LVIS, which dilates to $5.7 \mathrm{~mm}$ in maximum diameter. An LVIS whose diameter was larger than that of the parent blood vessel was protruded into the aneurysmal neck in a barrel-like shape, but the stent could not cover the origin of the branch vessel to prevent the coil from filling the origin. However, the LVIS protruding in a barrel-like shape may have reduced the width of the blood flow space of the SCA in comparison with a parent-artery-diameter-matched LVIS, facilitating the preparation of a framing coil involving preservation of the SCA (Fig. 4). Support by the stable framing coil and protruding LVIS facilitated dense intraaneurysmal coil embolization. Thus, even when an LVIS protruding into the aneurysmal neck does not completely cover the origin of a branch vessel, a reduction in the width of the blood flow space of the branch vessel may lead to 

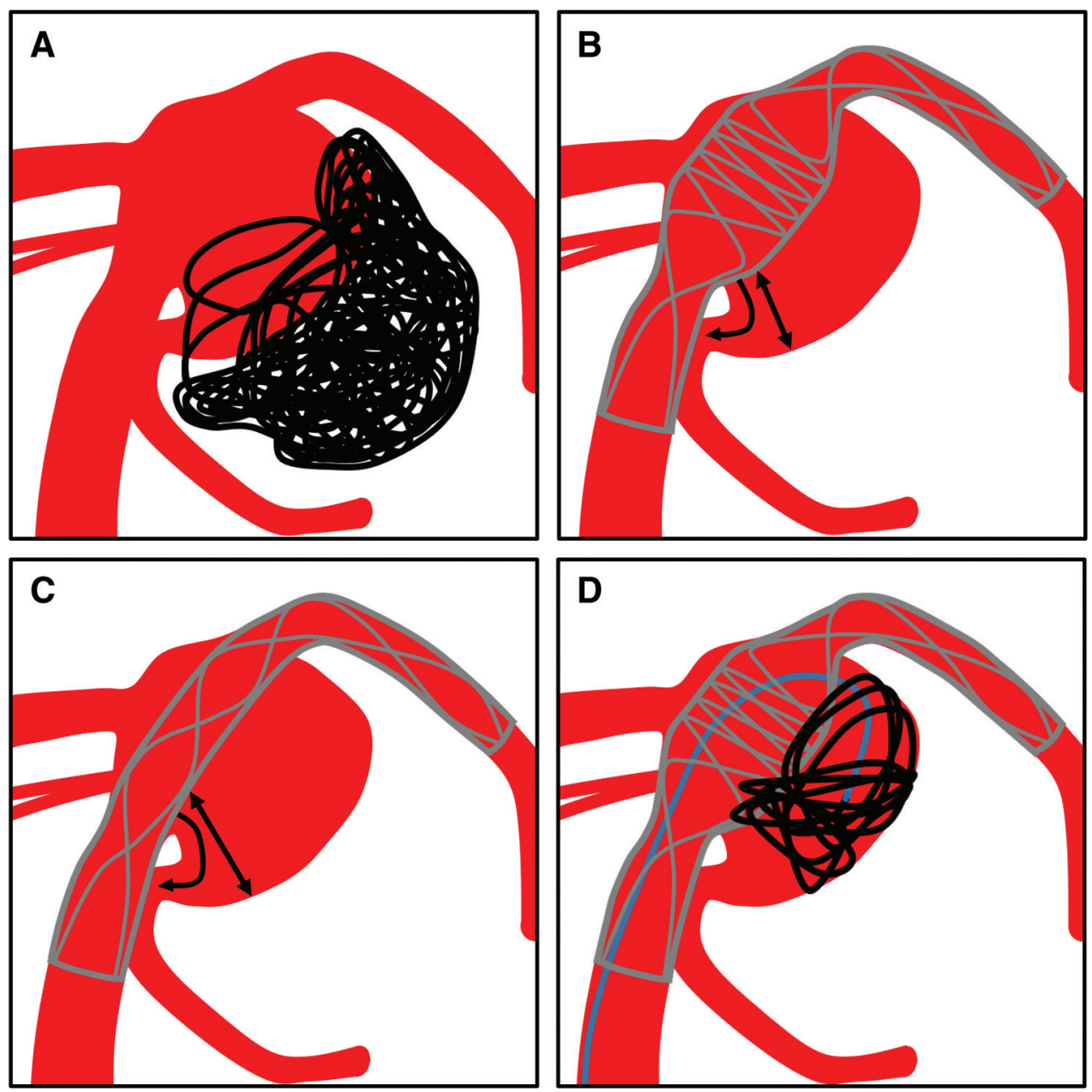

Fig. 4 Scheme of LVIS deployment. (A) Cerebral angiography before treatment showed a coil mass on initial treatment, a recurrent aneurysm, and the left SCA branching from the dome. (B and C) The LVIS protruded into the aneurysmal neck in a barrel-like shape and was deployed (B). The LVIS was deployed according to the diameter of a parent blood vessel (C). Left SCA blood flow (arrow). Width of the blood flow space of the left SCA (both arrows). (D) The LVIS was protruded into the aneurysmal neck in a barrel-like shape and deployed. This reduced the width of the blood flow space of the left SCA, facilitating the preparation of a framing coil involving preservation of the SCA. LVIS: low-profile visualized intraluminal support device; SCA: superior cerebellar artery

preservation of the branch vessel and dense intra-aneurysmal coil embolization.

An LVIS may prevent coil deviation to an unnecessary area and occlude aneurysms through flow-diverting effects. ${ }^{10-13)}$ In the barrel-like area of an LVIS dilated to a maximum diameter, the metal coverage rate is higher than in the LVIS-dilation-restricted area. The metal coverage rate is increased by adding "system-push" operations on LVIS deployment, and that of an LVIS dilated to a maximum diameter further increases with an increase in the stent diameter. ${ }^{10)}$ Furthermore, the metal coverage rate on the lesser curvature side of an LVIS is relatively higher than on its greater curvature side. ${ }^{10)}$ Therefore, in the present case, the stent was able to be deployed such that a high metal coverage rate was obtained at the aneurysmal neck, and the flow-diverting effects of the LVIS may have helped to inhibit aneurysmal enlargement. In addition, the barrel-like area with a high metal coverage rate prevented coil deviation to the stent lumen on intra-aneurysmal coil filling, leading to dense coil embolization of the aneurysm.

Flow diverters have been routinely used for the treatment of intracranial aneurysms, but the risk of ischemic complications related to posterior circulation aneurysms is relatively higher than that related to anterior circulation aneurysms..$^{14,15)}$ Therefore, concerning posterior circulation aneurysms, treatment using a flow diverter is proposed for a limited number of patients with a poor clinical course in whom no other treatment method could be performed.$^{15)}$ The presence 
of a branch vessel from an aneurysm is a factor for incomplete occlusion after flow-diverter deployment. In particular, a branch vessel with no sufficient collateral pathway may be patent even after flow-diverter deployment, resulting in unsuccessful aneurysm occlusion. ${ }^{16,17)}$ In addition, many wide-necked aneurysms require additional treatment after flow-diverter deployment, and it is suggested that a specific time is required for endothelialization of a wide neck. ${ }^{18)}$ Therefore, in the present case, treatment by flow-diverter deployment alone was not considered as an option.

If embolization with two microcatheters ${ }^{19}$ ) had been possible in the present case, denser coil embolization of the aneurysm may have been achieved. However, when a guiding catheter was guided into the left vertebral artery, blood flow stasis of this artery was noted and catheter insertion into the right vertebral artery was not conducted considering the risk of ischemic complications. Furthermore, if the semi-jailing technique ${ }^{20)}$ had been adopted, the position of a coil to be inserted into the aneurysm may have been more readily adjusted. However, in the present case, a microcatheter was guided from the inflow zone to the outflow zone and the effects of the semi-jailing technique were suspected to be limited. In addition, we wanted to perform coil embolization after confirming the relationship between the completely deployed LVIS and SCA; therefore, coil embolization was performed using the jailing technique. Dense coil embolization of the inflow zone of the aneurysm with the microcatheter pushed back was conducted, and intra-aneurysmal blood flow was maintained in the outflow zone where SCA blood flow was present; therefore, aneurysmal enlargement may be inhibited for a long period. A previous study found that the flow diverting effects of double LVIS usage were more marked than those of a single flow diverter. ${ }^{13)}$ If aneurysmal enlargement is observed during long-term follow-up in the present case, an additional LVIS will be deployed.

\section{Conclusion}

We reported a patient in whom dense coil embolization was performed while preserving a branch vessel from the dome under support by an LVIS protruded into the aneurysmal neck in a barrel-like shape such that the metal coverage rate was high, leading to a favorable embolic state. Even if an LVIS does not completely cover the origin of a branch vessel, a reduction in the width of the blood flow space of the branch vessel may aid in preservation of the branch vessel and dense coil embolization.

\section{Informed Consent}

Written informed consent regarding the publication of this case report was received from the patient.

\section{Disclosure Statement}

The authors declare no conflict of interest.

\section{References}

1) Du EHY, Shankar JJS: LVIS Jr 'shelf' technique: an alternative to Y stent-assisted aneurysm coiling. J Neurointerv Surg 2016; 8: 1256-1259.

2) Darflinger RJ, Chao K: Using the barrel technique with the LVIS Jr (low-profile visualized intraluminal support) stent to treat a wide neck MCA bifurcation aneurysm. $J$ Vas Interv Neurol 2015; 8: 25-27.

3) Inoue A, Tagawa $M$, Matsumoto $S$, et al: Utility of bulging technique for endovascular treatment of small and widenecked aneurysms with a low-profile visualized intraluminal support (LVIS Jr.) device: a case report and review of the literature. Interv Neuroradiol 2018; 24: 125-129.

4) Ohshima $T$, Miyachi $S$, Matsuo N, et al: Novel vertebral artery flow reversal method for preventing ischemic complication during endovascular intervention. J Stroke Cerebrovasc Dis 2018; 27: e144-e147.

5) Gao X, Liang G, Li Z, et al: A single-centre experience and follow-up of patients with endovascular coiling of large and giant intracranial aneurysms with parent artery preservation. J Clin Neurosci 2012; 19: 364-369.

6) Akgul E, Aksungur E, Balli T, et al: Y-stent-assisted coil embolization of wide-neck intracranial aneurysms. A single center experience. Interv Neuroradiol 2011; 17: $36-48$

7) Brassel F, Melber K, Schlunz-Hendann M, et al: Kissing-Y stenting for endovascular treatment of complex wide necked bifurcation aneurysms using Acandis Acclino stents: results and literature review. $J$ Neurointerv Surg 2016; 8: 386-395.

8) Aydin K, Stracke CP, Barburoglu M, et al: Long-term outcomes of wide-necked intracranial bifurcation aneurysms treated with T-stent-assisted coiling. J Neurosurg 2019; 6: 1-10.

9) Miyachi S, Matsubara N, Izumi T, et al: Stent/balloon combination assist technique for wide-necked basilar terminal aneurysms. Interv Neuroradiol 2013; 19: 299-305.

10) Matsuda Y, Chung J, Keigher K, et al: A comparison between the new low-profile visualized intraluminal support (LVIS Blue) stent and the flow redirection endoluminal device (FRED) in bench-top and cadaver studies. J Neurointerv Surg 2018; 10: 274-278. 
11) Dholakia R, Sadasivan C, Fiorella DJ, et al: Hemodynamics of flow diverters. $J$ Biomech Eng 2017; 139. doi: 10.1115/1.4034932.

12) Koch MJ, Stapleton CJ, Raymond SB, et al: LVIS Blue as a low porosity stent and coil adjuvant. J Neurointerv Surg 2018; 10: 682-686.

13) Wang C, Tian Z, Liu J, et al: Flow diverter effect of LVIS stent on cerebral aneurysm hemodynamics: a comparison with Enterprise stents and the Pipeline device. J Transl Med 2016; 14: 199.

14) Brinjikji W, Murad MH, Lanzino G, et al: Endovascular treatment of intracranial aneurysms with flow diverters: a meta-analysis. Stroke 2013; 44: 442-447.

15) Wang CB, Shi WW, Zhang GX, et al: Flow diverter treatment of posterior circulation aneurysms. A meta-analysis. Neuroradiology 2016; 58: 391-400.

16) Kan P, Duckworth E, Puri A, et al: Treatment failure of fetal posterior communicating artery aneurysms with the pipeline embolization device. J Neurointerv Surg 2016; 8: 945-948.

17) Moshayedi H, Omofoye OA, Yap E, et al: Factors affecting the obliteration rate of intracranial aneurysms treated with a single pipeline embolization device. World Neurosurg 2017; 104: 205-212.

18) Dmytriw AA, Adeeb N, Kumar A, et al: Flow diversion for the treatment of basilar apex aneurysms. Neurosurgery 2018; 83: 1298-1305.

19) Kwon OK, Kim SH, Kwon BJ, et al: Endovascular treatment of wide-necked aneurysms by using two microcatheters: techniques and outcomes in 25 patients. AJNR Am J Neuroradiol 2005; 26: 894-900.

20) Hong B, Patel NV, Gounis MJ, et al: Semi-jailing technique for coil embolization of complex, wide-necked intracranial aneurysms. Neurosurgery 2009; 65: 1131-1138; discussion 1138-1139. 